\title{
Barium Salts should not be Included in the Digitalis Group.
}

\author{
By \\ KEISUKE YAMANOUCHI. \\ (山内 惠 助) \\ (From the Pharmacological Laboratory of Prof. S. Yagi, \\ Tohoku Imperial University, Sendai.)
}

On the ground of their resemblance to digitalis bodies in their action on the heart and the blood vessels barium salts, for instance barium chloride, have been counted among the digitalis group in most of the textbooks. But the resemblance in their action would seem to be only superficial, when the reports relating to the study of this matter were looked over. An attempt has been made therefore in the present research to ascertain whether barium salts ought to belong to the digitalis group, subjecting the heart and the vessels of the frog to the action of barium chloride.

\section{Action on the Heart.}

With regard to the observations of the action of barium chloride upon the frog's heart, Boeh $\mathrm{m}^{1)}$ states that by this drug injected into the lymph sack of the animal the contraction of the auricle and the ventricle is rendered stronger, and their relaxation then less and less until the heart is led to a systolic arrest, this feature falling in perfectly with that in the case of digitalis; Poulsson ${ }^{2}$ also concludes that on the excised frog's heart barium chloride causes a systolic standstill by its intracardiac, and a diastolic pause by its exocardiac administration, and there exists an analogous, but not so regular and constant difference in the action, as observed in the case of digitalis bodies; Werschinin $n^{3)}$ claims that under barium chloride the excised heart of the frog undergoes changes similar to those induced by the members of digitalis series, resulting in a standstill in systole or in diastole according to its dosage.

These effects of causing an increased systole followed by an incomplete diastole, finally bringing about a systolic arrest, coincide with those mani- 
fested by larger doses of digitalis bodies. But smaller quantities of digitalis bodies increase not only the systolic but also the diastolic excursions, and this is said to be one of the peculiar properties by which all the members of this group are distinguished from other substances; accordingly barium must partake of this property in order to be included in the digitalis group. The literature concerning this subject, however, does not show plainly whether this drug can give rise to this characteristic change in smaller doses or not.

According to Straub's method, I have observed the effect upon the ventricle beats of the excised heart of the rana japonica, which is quite like the rana temporaria not only in its appearance but also in its pharmacological reaction, after the administration of barium chloride in various concentrations. When, by adding the solution of this drug to the perfusion liquid, its concentration is made 0.05 per cent, the ventricle becomes immediately stronger in systole, while the extent of its dilation goes on diminishing until the ventricle ceases to relax, remaining in the state of extreme systole, as seen in Fig. 1. The amplitude of the ventricle beats

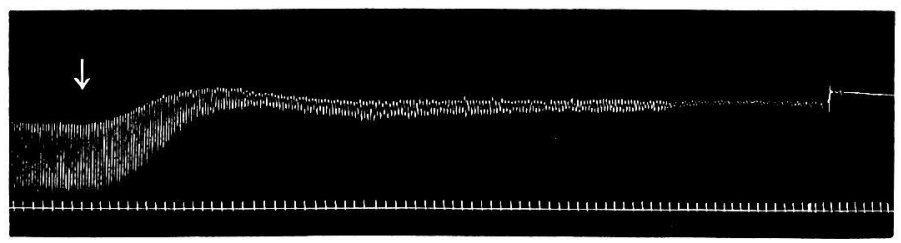

Fig. 1. Effect of barium chloride upon the excised heart of a rana japonica. At the arrow $0.001 \mathrm{grm}$. is added to the perfusion liquid (2.0 c.c.). Time in 5 seconds.

is rendered shorter than usual, though the contraction is augmented, for the decrease of the diastolic excursions is greater in degree compared with the increase of the systolic. But only when the systolic contraction predominates at the beginning, while the decrease in diastole is less developed, the amplitude is sometimes found to be increased temporarily. These features resemble so much those under larger quantities of digitalis bodies, that they can be hardly distinguished from them in this respect. When the amount of barium chloride to be added to the perfusion liquid is diminished, the same changes in character can be observed distinctly, even though they are inferior in degree to those above mentioned. Since, however, these effects in this case are not so marked-in degree, it follows that the initial increase of the amplitude arises more frequently and continues longer, 
and the heart does not easily pass into the systolic standstill. Even in a minimum effective concentration this drug never tends to dilate more fully than usual in diastole. Fig. 2 will serve to illustrate these pheno-

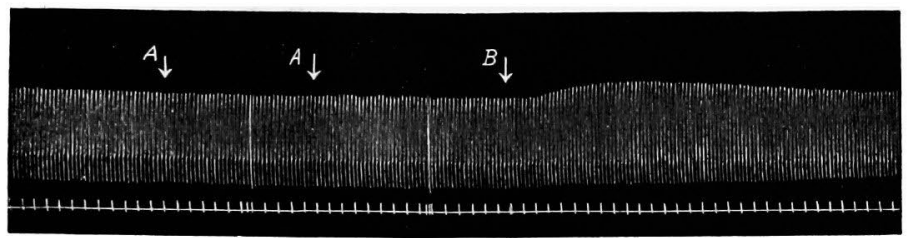

Fig. 2. Effect of barium chloride upon the excised heart of a rana japonica. At each A $0.000005 \mathrm{grm}$., at B $0.00001 \mathrm{grm}$. are added to the perfusion liquid ( 2.0 c.c.). Time in 5 seconds.

mena : By 0.0005 per cent barium chloride solution the ventricle beats are by no means affected, and when the concentration is made amounting to 0.001 per cent, they succumb for the first time to the alterations described previously.

In view of these facts, it should be assumed that barium salts do not possess an action of perfecting the relaxation of the ventricle even in weak concentrations, as digitalis and its allies do.

\section{Action on the Blood Vessels.}

It is almost generally acknowledged that barium salts have a tendency to constrict the vessels in various regions of the body acting on their muscle directly, just as digitalis bodies do. The constrictor effect of the latter, however, is limited only in higher concentrations, and in lower ones, on the contrary, they are apt to dilate the vessels, even though, of course, the susceptibility of the vessels to these drugs varies considerably according to the regions to which the vessels belong. I will now try to investigate whether barium exerts the dilatator effect on the vessels.

According to Saito, ${ }^{4)}$ the femoral vessels of the frog would seem to be dilated by lower concentrations of digitalis bodies and constricted by higher ones. If this be really the case, employing the femoral vessels is very favourable for this purpose. The thigh of a rana japonica was first perfused by Ringer's solution according to Trendelenburg's method, and the solution was then substituted by that containing barium chloride, and thus the effect of this drug upon the amount of the outflow was observed. The amount of the outflow, of course, has a close relation to the 
pressure of the perfused liquid; the following procedure was, therefore, employed to prevent as much as possible, the varying of the pressure which is liable to occur when Ringer's solution is substituted by that containing the agent. The perfusion apparatus consisted of a Mariotte's flask containing Ringer's solution, an artery cannula, and the rubber tubing connecting them. The last was divided into two ways in certain length by means of a glass $\mathrm{Y}$-tube and a three-way cock. One of the two tubes was closed previously by the cock and had barium chloride in Ringer's solution injected, one-third as much as its capacity, through its wall at the distal end by means of a syringe, driving away the Ringer's solution which had been there, and the cannula was then inserted into the aorta. When the amount of the outflow of Ringer's solution per minute had become constant, the solution containing barium chloride was allowed, by turning the cock, to take the place of the former.

By the employment of this method, strophanthin was found to cause a clear increase in the amount of the outflow in 0.005 per cent, an increase followed by a decrease in 0.05 per cent, and a distinct decrease from the first in 0.1 per cent solution. These facts indicate that this method can be used for the determination of the above mentioned subject, agreeing with Saito's report, although there is more or less difference in their effective doses, which may probably be attributed to a variation of the sensibility of the animal due to the season. Then this method was applied in order to observe the effect of barium chloride upon the vessels. After the administration of 0.0001 per cent solution perceptible changes in the drops did not follow within thirty minutes, but a marked decrease occurred under 0.01 per cent, while in the middle concentration, 0.001 per cent, a decrease was observed in a small degree, and in 0.0005 per cent very slightly (Fig. 3).

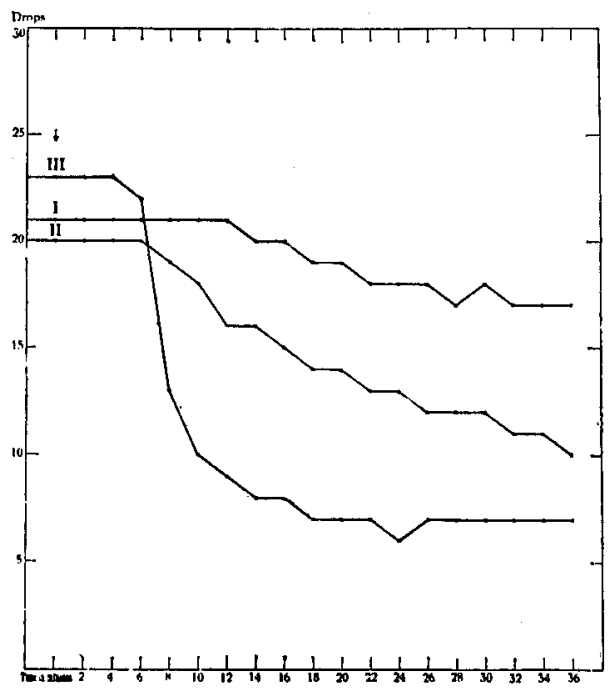

Fig. 3. Effect of barium chloride on the blood vessels of a rana japonica. (I) $0.0005 \%$, (II) $0.001 \%$, (III) $0.01 \%$ solution. 
Based on the results obtained from these experiments, it must be claimed that barium chloride always tends to act on the vessels constrictively, if it is only effective, regardless of its concentration, and the dilator effect by no means follows after its administration. Such being the case, barium salts should be said to differ from all the members of the digitalis group also in this respect.

Thus, even though the action of barium salts on the heart and the blood vessels, at least on the frog's organs, presents some points of close resemblance to that of digitalis bodies, yet it would not be reasonable to include this drug in the digitalis group, for it does not possess the most important property by which the digitalis group is characterized.

\section{Bibliography.}

(1) Boehin, Arch. f. exp. Path. u. Pharm., 1875, 3, 216.

(2) Poulsson, ib. 1910, 62, 365.

(3) Werschinin, ib. 1911, 66, 191.

(4) Saito, Kyoto Igaku Zasshi, 1923, 20, 450. 